\title{
LC-MS/MS METHOD DEVELOPMENT AND VALIDATION FOR THE DETERMINATION OF CARDIAZOL IN HUMAN PLASMA
}

\section{IRYNA DRAPAK ${ }^{1}$, BORYS ZIMENKOVSKY², LINA PEREKHODA ${ }^{3}$, SERGIY KOVALENKO4 ${ }^{4}$, LILIYA LOGOYDA5*}

${ }^{1}$ General, Bioinorganic, Physical and Colloidal Chemistry Department, Danylo Halytsky Lviv National Medical University, Lviv City, Ukraine, ${ }^{2}$ Pharmaceutical, Organic and Bioorganic Chemistry Department, Danylo Halytsky Lviv National Medical University, Lviv City, Ukraine, ${ }^{3}$ Medicinal Chemistry Department, National University of Pharmacy, Kharkiv City, Ukraine, ${ }^{4}$ Organic and Bioorganic Chemistry Department, Zaporizhzhya State Medical University, Zaporizhzhya City, Ukraine, ${ }^{5}$ Pharmaceutical Chemistry Department, Pharmaceutical Faculty, I. Horbachevsky Ternopil State Medical University, Ternopil City, Ukraine Email: logojda@tdmu.edu.ua

Received: 06 Apr 2019, Revised and Accepted: 01 Jun 2019

\section{ABSTRACT}

Objective: The main purpose of this study was to develop a simple, precise, rapid and accurate method for the quantification of cardiazol in human plasma.

Methods: Chromatography was achieved on Discovery C18, $50 \times 2.1 \mathrm{~mm}, 5 \mu \mathrm{m}$ column. Samples were chromatographed in a gradient mode (eluent A (acetonitrile-water-formic acid, 5: 95: $0.1 \mathrm{v} / \mathrm{v}$ ), eluent B (acetonitrile-formic acid, 100: $0.1 \mathrm{v} / \mathrm{v}$ )). The initial content of the eluent B of 8\%, which increases linearly to $1.0 \mathrm{~min}$ to $100 \%$, is maintained up to $1.5 \mathrm{~min}$ and returned to the original $8 \%$ to $1.51 \mathrm{~min}$. The mobile phase was delivered at a flow rate of $0.400 \mathrm{ml} / \mathrm{min}$ into the mass spectrometer ESI chamber. The sample volume was $300 \mu \mathrm{l}$.

Results: The total chromatographic run time was $2.5 \mathrm{~min}$ and the elution of cardiazol and IS (difenoconazole) occurred at $\sim 2.15$ and $1.98 \mathrm{~min}$, respectively. A linear response function was established at $1-100 \mathrm{ng} / \mathrm{ml}$ for cardiazol and difenoconazole in human plasma. The $\%$ mean recovery for cardiazol in LQC, MQC and HQC was $102.8 \%, 100.3 \%$ and $95.9 \%$. The lowest concentration with the RSD $<20 \%$ was taken as LLOQ and was found to be $1.10 \mathrm{ng} / \mathrm{ml}$ for cardiazol. The \% accuracy of LLOQ samples prepared with the different biological matrix lots was found $109.7 \%$, which were found within the range of $80.00-120.00 \%$ for the seven different plasma lots. \% CV for LLOQ samples was observed as $11.9 \%$, which are within $20.00 \%$ of the acceptance criteria.

The within-run coefficients of variation ranged between $0.311 \%$ and $0.601 \%$ for cardiazol. The within-run percentages of nominal concentrations ranged between $99.80 \%$ and $100.41 \%$ for cardiazol. The between-run coefficients of variation ranged between $0.332 \%$ and $0.615 \%$ for cardiazol. The between-run percentages of nominal concentrations ranged between $98.18 \%$ and $101.21 \%$ for cardiazol.

Conclusion: A rapid method was developed for simultaneous determination of cardiazol in human plasma. The method was strictly validated according to the ICH guidelines. Acquired results demonstrate that the proposed strategy can be effortlessly and advantageously applied for routine examination of cardiazol in human plasma.

Keywords: LC-MS/MS, Cardiazol, Human plasma, Validation, Pharmacokinetic studies

(C) 2019 The Authors. Published by Innovare Academic Sciences Pvt Ltd. This is an open access article under the CC BY license (http://creativecommons.org/licenses/by/4.0/) DOI: http://dx.doi.org/10.22159/ijap.2019v11i4.33482

\section{INTRODUCTION}

Bioanalytical methodology is the founder of the study of bioequivalence and therapeutic drug monitoring, so the main focus in the study of pharmacokinetics and bioequivalence should be given to the development of bioanalytical methods of analysis. Bioanalytical method validation employed for the quantitative determination of drugs and their metabolites in biological fluids plays a significant role in the evaluation and interpretation of bioavailability, bioequivalence, pharmacokinetic, and toxicokinetic study data [1]. These studies generally support regulatory filings. The quality of these studies is directly related to the quality of the underlying bioanalytical data.

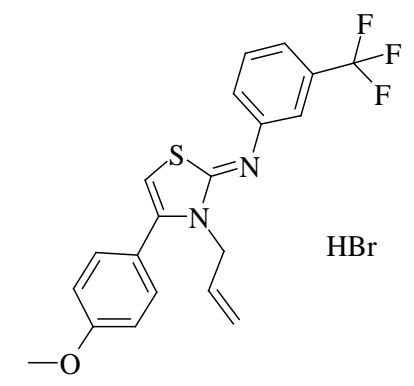

Fig. 1: Chemical structure of cardiazol
Cardiazol (fig. 1) (hydrobromide [3-Allyl-4-(41-methoxyphenyl)-3Hthiazole-2-ylidene]-(32-trifluoromethylphenyl) amine)-the original newly synthesized cardioprotective, which, in addition to the cardioprotective effect, also exhibits hypolipidemic, antiinflammatory, analgesic and antioxidant activity [2]. This indicates the promise of this substance for further preclinical studies.

Therefore, it was thought desirable to develop a simple, accurate, cheap and fast procedure that could be applied for the determination of cardiazol in human plasma, this study performed assay validations as per guidelines [3-5]. While this method with validation details was economical and applied for pharmacokinetic studies of cardiazol.

\section{MATERIALS AND METHODS}

\section{Chemicals and reagents}

Cardiazol (purity $98.5 \%$ ) was synthesized by us, difenoconazole (Internal Standard) (purity $98.8 \%$ ) was purchased from Moehs Catalana, S. L., Spain, Zhejiang Huahai Pharmaceutical Co., Ltd, KHP, EDQM-Council of Europe. HPLC grade acetonitrile and methanol were purchased from CHROMASOLV, HPLC grade formic acid was purchased from Fluka. All other chemicals and reagents were of analytical grade. Microcaps ${ }^{\circledR}$ disposable micropipettes $(50 \mu \mathrm{l}$, catalog number: 1-000-0500) were purchased from Drummond Scientific Company, USA. The control of human dipotassium ethylenediaminetetraacetic acid $\left(\mathrm{K}_{2}\right.$ EDTA) plasma sample was procured from Red Cross Society, Ukraine. 


\section{Instrumentation and chromatographic conditions}

A Shimadzu HT (Shimadzu, Japan) LC system equipped with degasser (DGU-14A), binary pump (LC-20ADXR) along with autosampler (SIL-20AC HT) was used to inject $5 \mu \mathrm{l}$ aliquots of the processed samples on Discovery C18, $50 \times 2.1 \mathrm{~mm}, 5 \mu \mathrm{m}$ column maintained at $25 \pm 1^{\circ} \mathrm{C}$. Samples were chromatographed in a gradient mode (eluent A (acetonitrile-water-formic acid, 5: 95: $0.1 \mathrm{v} / \mathrm{v}$ ), eluent B (acetonitrile-formic acid, 100: $0.1 \mathrm{v} / \mathrm{v}$ )). Samples were chromatographed in gradient mode. The initial content of the eluent $\mathrm{B}$ of $8 \%$, which increases linearly to $1.0 \mathrm{~min}$ to $100 \%$, is maintained up to $1.5 \mathrm{~min}$ and returned to the original $8 \%$ to $1.51 \mathrm{~min}$. The mobile phase was delivered at a flow rate of $0.400 \mathrm{ml} / \mathrm{min}$ into the mass spectrometer ESI chamber. Parameters of electrospray ionizer and MRM parameters are listed in table 1-2. The analytical data were processed by Analyst Software (version 1.5.2).

Table 1: Parameters of ionizer electrospray

\begin{tabular}{lll}
\hline S. No. & Parameter & Value \\
\hline 1 & Polarity & Positive \\
2 & Nebulizer Gas (NEB, Gas 1) & 15 \\
3 & Curtain Gas (CUR) & 8 \\
4 & Collision Gas (CAD) & 4 \\
5 & IonSpray Voltage (IS) & 5000 \\
6 & Temperature (TEM) & 400 \\
7 & Turbo IonSpray Gas & 8 \\
8 & Horizontal Position & 8.0 \\
9 & Lateral Position & 2.0 \\
\hline
\end{tabular}

Table 2: Multiple reaction monitoring (MRM) parameters

\begin{tabular}{|c|c|c|c|c|c|c|c|}
\hline ID & Parent, $\mathbf{m} / \mathbf{z}$ & Daughter, m/z & Time, ms & DP, V & EP, V & $\mathrm{CE}, \mathrm{V}$ & CXP, V \\
\hline Cardiazol & 391.018 & 360.1 & 40 & 46 & 11 & 35 & 20 \\
\hline Difenoconazole & 406.100 & 251.2 & 40 & 61 & 11 & 35 & 20 \\
\hline
\end{tabular}

*Abbreviations: DP, declustering potential; FP, focusing potential; EP, entrance potential; CE, collision energy; CXP, collision cell exit potential

\section{Standard solutions}

Cardiazil and IS were weighed accurately into volumetric flasks using an analytical microbalance. Approximately $1 \mathrm{mg} / \mathrm{ml}$ primary stock solutions of cardiazol and $1 \mathrm{mg} / \mathrm{ml}$ primary stock solutions of difenoconazole (IS) solutions were prepared in methanol. The stock solutions were stored at $-20^{\circ} \mathrm{C}$, which was found to be stable for $1 \mathrm{mo}$. The stock solutions of cardiazol and difenoconazole were successively diluted with methanol and water to prepare secondary stocks and working solutions. Secondary stock solutions and working solutions were used to prepare calibration curve (CC) and quality control (QC) samples. Working stock solutions were stored at $4{ }^{\circ} \mathrm{C}$ for a week. Working stocks were used to prepare plasma calibration standards. A working IS solution $(50 \mathrm{ng} / \mathrm{ml}$ ) was prepared in acetonitrile: methanol $(50: 50 \mathrm{v} / \mathrm{v})$. Blank human plasma was screened before spiking to ensure that it was free from endogenous interference at retention times of cardiazol and difenoconazole, respectively. Calibration standards' samples $(1-100 \mathrm{ng} / \mathrm{ml}$ for cardiazol and difenoconazole) were prepared by spiking the blank human $\mathrm{K}_{2}$ EDTA plasma with appropriate concentration of cardiazol.

Samples for the determination of precision and accuracy were prepared by spiking control human plasma in bulk with cardiazol at appropriate concentrations (for cardiazol $3.00 \mathrm{ng} / \mathrm{ml}$ low QC [LQC], $30.00 \mathrm{ng} / \mathrm{ml}$ medium QC [MQC], and $75.00 \mathrm{ng} / \mathrm{ml}$ high QC [HQC]) and $120 \mu \mathrm{L}$ plasma aliquots were distributed into different tubes. All the samples were stored at $-80^{\circ} \mathrm{C} \pm 10^{\circ} \mathrm{C}$.

\section{Sample preparation}

A simple protein precipitation extraction method was followed for extraction of cardiazol at from human plasma. From the deep freezer, the required quantities of CC standards and QC samples were withdrawn. The samples were thawed at room temperature. To an aliquot of $100 \mu \mathrm{l}$ plasma, $20 \mu \mathrm{l}$ of IS was added. To this mixture, $300 \mu \mathrm{l}$ of acetonitrile: methanol $(50: 50 \mathrm{v} / \mathrm{v})$ was added and vortexed for $2 \mathrm{~min}$, followed by centrifugation at $6000 \mathrm{rpm}$ for $5 \mathrm{~min}$ at $4{ }^{\circ} \mathrm{C}$. After centrifugation, approximately $50 \mu \mathrm{l}$ supernatant was aliquoted into, respectively, labeled autosampler vials, which were later placed in the autosampler at $15{ }^{\circ} \mathrm{C} \pm 4{ }^{\circ} \mathrm{C}$. $10 \mu \mathrm{l}$ of the sample was injected onto LC-MS/MS system for analysis.

\section{Method validation}

A full validation according to the ICH guidelines was performed for the assay in $\mathrm{K}_{2}$ EDTA human plasma.

\section{Specificity and selectivity}

The specificity of the method was evaluated by analyzing human plasma samples from different lots to investigate the potential interferences at the chromatographic peak region for cardiazol and IS. The acceptance criterion for the experiment was that should have $<20 \%$ area response to that of the LLOQ level response in the same matrix. Two lots of hemolyzed plasma samples were also analyzed to ensure specificity against potential biological interferences.

\section{Linearity}

The points CC $(1-100 \mathrm{ng} / \mathrm{ml})$ were constructed by plotting the peak area ratio of analyte: IS against the nominal concentration of calibration standards in $\mathrm{K}_{2}$ EDTA human plasma. Following the evaluation of different weighting factors, the results were fit into linear regression analysis using $1 / \mathrm{X} 2$ (X: Concentration) weighing factor. The CC should have a correlation coefficient (r) of 0.99 or better. The acceptance criteria for each back-calculated standard concentration were $\pm 15 \%$ deviation from the nominal value except at LLOQ, which was set at $\pm 20 \%$.

\section{Recovery}

The efficiency of cardiazol and IS extraction from human plasma was determined by comparing the responses of the analytes extracted from replicate QC samples $(n=6)$ with those of neat standard solutions spiked in post-extracted plasma blank sample at equivalent concentrations by protein precipitation extraction method. Recovery of cardiazol was determined at LQC $(3.00 \mathrm{ng} / \mathrm{ml})$ and HQC $(75.00 \mathrm{ng} / \mathrm{ml})$ concentrations, whereas the recovery of IS was determined at a single concentration of $20 \mathrm{ng} / \mathrm{ml}$.

\section{Matrix effect}

The effect of human plasma constituents over the ionization of cardiazol and IS was determined by post-column infusion method to evaluate matrix effect. Briefly, an infusion pump delivers a constant amount of analyte into LC system outlet entering to mass spectrometer inlet. To follow the analyte signal, the mass spectrometer was operated in MRM mode. The human plasma constituent sample extract was injected on LC column. A steady ion response was obtained as a function of time since the analyte was infused at a constant rate. Any endogenous compound that elutes from the column which causes a variation in ESI response of the infused analyte was seen as suppression or enhancement in the 
response of the infused analyte. A separate experiment was performed with cardiazol and IS solutions, which were infused at a constant rate, and blank matrix sample injected through the LC. To evaluate matrix effect, different lots of human plasma were spiked with analyte concentration levels at LQC and HQC levels. According to guidelines, the acceptance criterion for each back-calculated concentration was $\pm 15 \%$ deviation from the nominal value.

\section{Precision and accuracy}

The intra-assay precision and accuracy were estimated by analyzing six replicates containing cardiazol at four different QC levels in human plasma. The four-level QC samples on four different runs were performed to assess the interassay precision. The acceptance criteria for each back-calculated standard concentration were $85-115 \%$ accuracy from the nominal value except at LLOQ which was set at $80-120 \%$.

\section{Stability experiments}

Stability tests were conducted to evaluate the stability of cardiazol in plasma samples under different conditions. $8 \mathrm{~h}$ bench top stability, processed samples stability (autosampler stability for $26 \mathrm{~h}$ at $10^{\circ} \mathrm{C}$ ), three cycles of freeze-thaw stability, $30 \mathrm{~d}$ of long-term stability at $-80 \pm 10^{\circ} \mathrm{C}$ were performed at LQC and HQC levels using six replicates at each level. Samples were considered stable if assay values' acceptance criterion was of accuracy (i.e., $85-115 \%$ from fresh samples) and precision (i.e., $\pm 15 \%$ relative standard deviation [RSD]).

\section{RESULTS AND DISCUSSION}

Cardiazol is original newly synthesized cardioprotective. That's why previously was no any bioanalytical method development for cardiazol. But aim of our work was also to develop rapid, sensitive, and highly selective liquid chromatography-tandem mass spectrometry method for cardiazol and improve method development for all derivatives of thiazole.

In the present study, optimization and critical evaluation of mobile phase composition (gradient), flow rate, and analytical column were important to obtain a good resolution of peaks of interest from the endogenous components, which in turn affect reproducibility and sensitivity of the method. Selection of chromatographic conditions for the proposed method was optimized to suit the preclinical pharmacokinetic studies. To ease the sample preparation in microtubes and to reduce the usage of solvent, the plasma volume was kept low. Initial feasibility experiments of a various mixture(s) of solvents such as acetonitrile, methanol and formic acid along with altered flow rates (in the range of $0.1-0.6 \mathrm{ml} / \mathrm{min}$ ) were performed to optimize an effective chromatographic resolution of cardiazol and IS. Various analytical columns were tested to obtained good and reproducible response within the short run time. The resolution of peaks was best achieved with Discovery C18, $50 \times 2.1 \mathrm{~mm}, 5 \mu \mathrm{m}$ column. Samples were chromatographed in a gradient mode (eluent $\mathrm{A}$ (acetonitrile-water-formic acid, 5: 95: $0.1 \mathrm{v} / \mathrm{v}$ ), eluent B (acetonitrileformic acid, 100: $0.1 \mathrm{v} / \mathrm{v}$ )). The initial content of the eluent B of $8 \%$, which increases linearly to $1.0 \mathrm{~min}$ to $100 \%$, is maintained up to 1.5 min and returned to the original $8 \%$ to $1.51 \mathrm{~min}$. The mobile phase was delivered at a flow rate of $0.400 \mathrm{ml} / \mathrm{min}$ into the mass spectrometer ESI chamber. The injection volume was $300 \mu$ l.

The purpose of sample extraction optimization is mainly to achieve high extraction recovery with negligible or low matrix effects to improve the sensitivity and reliability of LC-MS/MS analysis [6-11]. A poor extraction procedure decreases method robustness due to the presence of endogenous interference in the sample extracts, which are not efficiently cleaned up due to poor extraction procedure decreases the method robustness due to the endogenous interference in the sample extracts. With time-saving advantage and simplicity, the protein precipitation extraction method was chosen as an extraction method. The attained LLOQ was sufficient to quantify cardiazol in low-dose pharmacokinetic studies.

Cardiazol eluted at $\sim 2.15$ min, respectively. During a direct infusion experiment, the mass spectra for cardiazol and IS revealed peaks at $\mathrm{m} / \mathrm{z}$ 391.08 and 406.100 , respectively as protonated molecular ions, $[\mathrm{M}+\mathrm{H}]+$. Typical multiple reaction monitoring chromatograms of cardiazol and internal standard in dipotassium ethylenediaminetetraacetic acid human blank plasma are shown in fig. 2.
Bl - CP (Blank) 391.0188350.100 Da - sample 6 of 38 from Lin CP.DU 4ul 060319.... Ares: 68 counts Height: $253 \mathrm{e}+001$ cps $R T$ : $217 \mathrm{~min}$

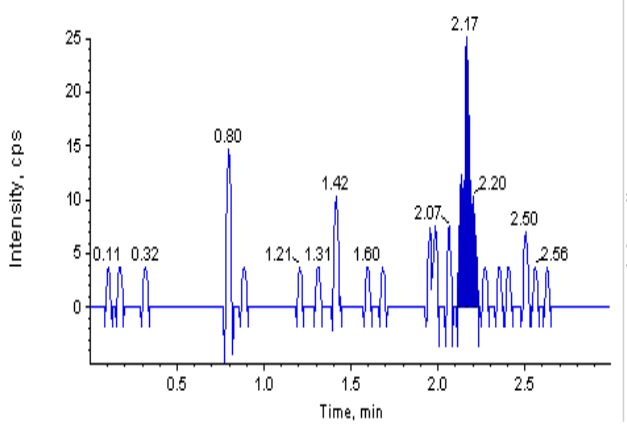

C1 - CP (Standard) $391.0183500 .100 \mathrm{Da} \cdot$ sample 8 of 38 from Lin CP.DU 4ul 060319.wiff Area: 1984 counts Height: $6.29 \mathrm{e}+002$ cps RT: 2.15 min

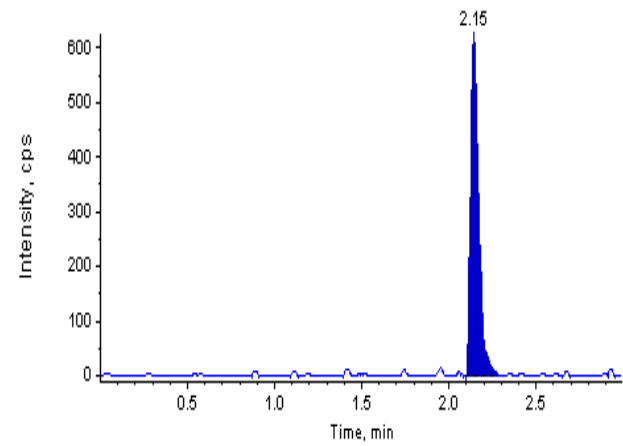

81. Dal(S)(Blank) 406.1002251.200 Da - sample 6 of 38 from Lin CP.DU 4ul 060319, with Area: 194 counts Height: $8.42 e+001$ cps RT: 1.99 min

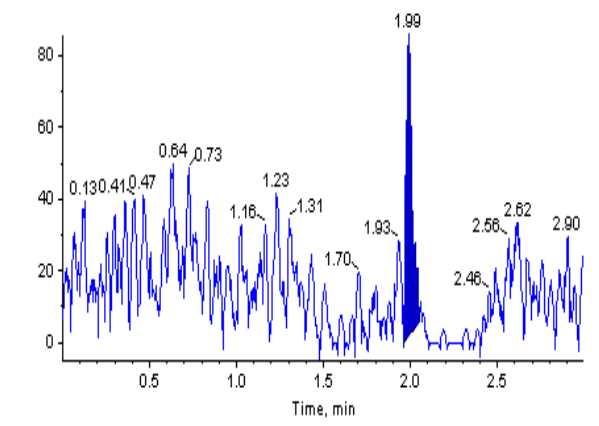

C1 - Da(1S)(Standard) 406.1002251.200 Da - sample 8 of 38 trom Lin CP.DU 4ul 060319.wif. Area: 140111 counts Height: $6.12 \mathrm{e}+004$ cps RT: 1.98 min

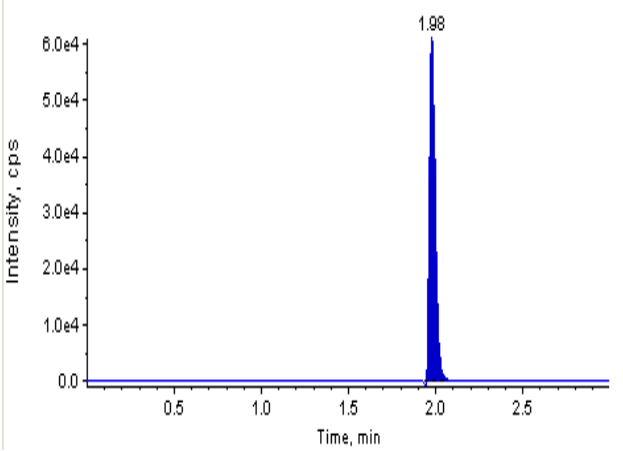

Fig. 2: Typical multiple reaction monitoring chromatograms of cardiazol (left) and internal standard (difenoconazole) (right) in dipotassium ethylenediaminetetraacetic acid human blank plasma 
The total chromatographic run time was $2.5 \mathrm{~min}$ and the elution of cardiazol and IS (difenoconazole) occurred at $\sim 2.15$ and $1.98 \mathrm{~min}$, respectively.

\section{Specificity}

Different lots of plasma were analysed to ensure that no endogenous interferences were present at the retention time of cardiazol LLOQ level samples along with plasma blank from the respective plasma lots were prepared and analysed (table 3).

\section{Linearity}

The calibration standard curves had a reliable reproducibility over the standard concentrations across the calibration range. The average regression $(n=3)$ was found to be $>0.99$ for an analyte.

Table 3: Results of specificity for cardiazol

\begin{tabular}{lllll}
\hline \multirow{2}{*}{ S. No. } & Enalapril & & & \\
\cline { 2 - 5 } & STD BL & LLOQ & \% Interference \\
\cline { 2 - 4 } & & Area & 2.15 & NIL \\
\hline 1 & 0 & 2031 & 2.14 & NIL \\
2 & 0 & 2033 & 2.15 & NIL \\
3 & 0 & 2029 & 2.14 & NIL \\
5 & 0 & 2038 & 2.14 & NIL \\
6 & 0 & 2032 & 2.14 & NIL \\
7 & 0 & 2030 & 2.15 & NIL \\
8 & 0 & 2042 & 2.15 & NIL \\
9 & 0 & 2029 & 2.15 & 2.15 \\
10 & 0 & 2031 & & \\
\hline
\end{tabular}

${ }^{*}$ Average of triplicate injections, In all plasma blanks, the response at the retention time of cardiazol was less than $20 \%$ of LLOQ response and at the retention time of IS, the response was less than $5 \%$ of mean IS response in LLOQ.

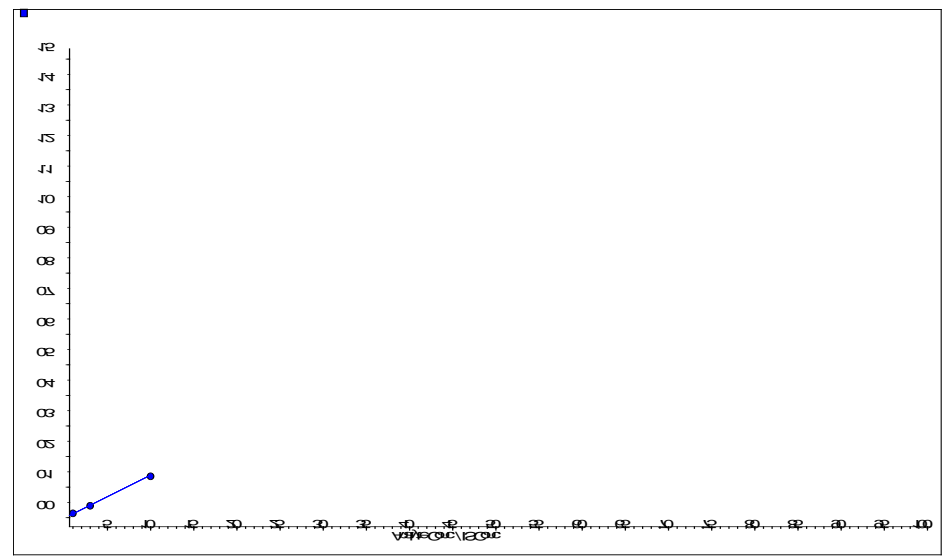

Fig. 3: The calibration curve of cardiazol in human plasma

The calibration curve (fig. 3) (peak area ratio Vs Concentration) was linear over working range for cardiazol of 1 to $100.00 \mathrm{ng} / \mathrm{ml}$ with 7 point calibration used for quantification by linear regression, shown in fig. 3. The regression equation for the analysis was $y=0.0141 x+-0.00146$ with coefficient of correction (r2) $=0.9985$.

\section{Recovery}

The \% mean recovery for cardiazol in LQC, MQC and HQC are listed in table 4.

Table 4: The \% mean recovery of cardiazol for LQC, MQC and HQC

\begin{tabular}{|c|c|c|c|}
\hline S. No. & LQC & MQC & HQC \\
\hline 1 & 2.97 & 28.4 & 76.6 \\
\hline 2 & 3.14 & 29.6 & 71.3 \\
\hline 3 & 3.10 & 31.5 & 70.6 \\
\hline 4 & 3.08 & 30.2 & 69.7 \\
\hline 5 & 3.13 & 30.7 & 71.3 \\
\hline Mean & 3.08 & 30.1 & 71.9 \\
\hline SD & 0.069 & 1.14 & 2.69 \\
\hline$\% \mathrm{CV}$ & 2.2 & 3.8 & 3.7 \\
\hline$\%$ Mean Recovery & 102.8 & 100.3 & 95.9 \\
\hline
\end{tabular}

*Abbreviations: Lower quality control (LQC), middle-quality control (MQC), higher quality control (HQC)

Each value is represented as a mean $\pm S D$ of 5 observations $(n=5)$, SD: Standard Deviation, RSD: Relative Standard Deviation, \#Acceptance criteria $<15 \%$.

The \% mean recovery for cardiazol in LQC, MQC and HQC was 102.8 $\%, 100.3 \%$ and $95.9 \%$.
Intraday (within run) and Inter-day (between run) precision and accuracy

The within-run coefficients of variation ranged between $0.311 \%$ and $0.601 \%$ for cardiazol. The within-run percentages of nominal concentrations ranged between $99.80 \%$ and $100.41 \%$ for cardiazol. The between-run coefficients of variation ranged between $0.332 \%$ and $0.615 \%$ for cardiazol. The between-run percentages of nominal 
Table 5: Intra-day and Inter-day precision data of cardiazol

\begin{tabular}{llll}
\hline Day & Intra-day precision & & Inter-day precision \\
\hline & Mean & RSD \% & Mean \\
\hline 1 & 99.80 & 0.378 & 101.21 \\
3 & 100.41 & 0.601 & 98.18 \\
RSD \% \\
\hline
\end{tabular}

*Each value is represented as a mean \pm SD of observations, SD: Standard Deviation, RSD: Relative Standard Deviation, \#Acceptance criteria<15 \%. The assay values on both the occasions (intra-and inter-day) were found to be within the accepted limits.

Table 6: Results of matrix effect of cardiazol

\begin{tabular}{ll}
\hline S. No. & LLQC \\
\hline 1 & 1.07 \\
2 & 1.15 \\
3 & 1.26 \\
4 & 1.10 \\
5 & 0.903 \\
Mean & 1.10 \\
SD & 0.130 \\
\% CV Mean Recovery & 11.9 \\
\hline
\end{tabular}

\section{Matrix effect}

The lowest concentration with the RSD $<20 \%$ was taken as LLOQ and was found to be $1.10 \mathrm{ng} / \mathrm{ml}$ for cardiazol. Results are presented in tables 6 .

*Abbreviations: Lower limit of quantification (LLOQ)

Each value is represented as a mean \pm SD of 5 observations $(n=5), S D$ : Standard Deviation, RSD: Relative Standard Deviation, \#Acceptance criteria $<20 \%$.
The \% accuracy of LLOQ samples prepared with the different biological matrix lots was found $109.7 \%$, which were found within the range of 80.00-120.00 \% for the seven different plasma lots. \% CV for LLOQ samples was observed as $11.9 \%$, which are within $20.00 \%$ of the acceptance criteria.

\section{Stability}

The predicted concentrations for cardiazol $(3.00 \mathrm{ng} / \mathrm{ml}$ and 75.00 $\mathrm{ng} / \mathrm{ml}$ ) deviated within $\pm 15 \%$ of the fresh sample concentrations in a battery of stability tests namely, in-injector $(22 \mathrm{~h})$, bench-top $(7 \mathrm{~h})$, and repeated four freeze/thaw cycles stability (table 7).

Table 7: Stability data of cardiazol at QCs in human plasma

\begin{tabular}{lllll}
\hline Nominal concentration (ng/ml) & Stability & Stability data & & \\
\cline { 3 - 5 } & & mean \pm SD ${ }^{\circ}(\mathbf{n}=6)$ & Accuracy (\%) & Precision (\% CV) \\
\hline Cardiazol-3.00 & 0 h & $3.10 \pm 0.43$ & 99.6 & 2.24 \\
& 7 h (bench-Top) & $3.01 \pm 0.37$ & 99.6 & 2.14 \\
& 22 h (in-injector) & $3.00 \pm 0.39$ & 99.3 & 2.26 \\
Cardiazol-75.00 & 3 FT cycles & $3.09 \pm 0.41$ & 99.9 & 2.16 \\
& 0 h & $75.2 \pm 0.39$ & 99.8 & 2.67 \\
& 7 h (bench-Top) & $75.4 \pm 0.21$ & 99.9 & 2.31 \\
& 22 h (in-injector) & $75.4 \pm 0.22$ & 99.9 & 3.06 \\
\hline
\end{tabular}

${ }^{\circ}$ Back-calculated plasma concentrations; $\bullet$ Mean assayed concentration/mean assayed concentration at $0 \mathrm{~h} \times 100$. FT: Freeze-thaw, SD: Standard deviation, QC: Quality control, The results were found to be within the assay variability limits during the entire process.

\section{CONCLUSION}

A highly sensitive, specific, reproducible, rapid and high-throughput LC-MS/MS assay was developed and validated to quantify cardiazol in human plasma as per the regulatory guidelines. The present method involved a simple precipitation method of sample preparation, which gave consistent and reproducible recoveries. Acquired results demonstrate that the proposed strategy can be effortlessly and advantageously applied for routine examination of cardiazol in human plasma. The combination was taken up for developing a bioanalytical method development and validation so that further it would be useful for performing pharmacokinetic studies.

\section{AUTHORS CONTRIBUTIONS}

All the authors have contributed equally

\section{CONFLICT OF INTERESTS}

The authors declare no conflict of interest

\section{REFERENCES}

1. https://www.omicsonline.org. [Last accessed on 01 May 2019]

2. Perekhoda LO, Drapak IV, Suleiman MM, Sych IA, Yaremenko VD. Synthesis and in silico research of derivatives of 3-allyl-4(R-phenyl)-N-(R1-phenyl)thiazole-2-imine. Der Pharma Chemica 2017;9:95-8.

3. Guideline on Bioanalytical method validation: European Medicines Agencies; 2011.

4. Sonawane LV, Poul BN, Usnale SV. Bioanalytical method validation and its pharmaceutical application a review. Pharm Anal Acta 2014;5:1-7.

5. Pushpa Latha E, Sailaja B. Bioanalytical method development and validation by HPLC: a review. J Appl Pharm 2014;1:1-9.

6. Liliya Logoyda. Bioanalytical method development and validation from the simultaneous determination of verapamil and enalapril in the present of enalaprilat by HPLC MS/MS. Int J Appl Pharm 2018;10:19-27. 
7. Liliya Logoyda. A HPLC-MS/MS method development and validation for the simultaneous determination of nifedipine and enalapril in human plasma. Int J Appl Pharm 2018;10:3542.

8. Ghosh C, Jain I, Shinde CP, Chakraborty BS. Rapid and sensitive liquid chromatography/tandem mass spectrometry method for simultaneous determination of enalapril and its major metabolite enalaprilat, in human plasma: application to a bioequivalence study. Drug Testing Anal 2012;4:94-103.

9. Logoyda L, Kondratova Y, Korobko D, Susla O, Soroka Y, Tsytsiura R, et al. Youden's test of the chromatographic determination of captopril in pharmaceuticals. Int J Green Pharm 2017;11:188-91.

10. Liliya Logoyda, Dmytro Korobko, Oleksandra Oleshchuk, Taras Proniv, Mariya Dmutriv. A HPLC MS/MS method development and validation for the simultaneous determination of bisoprolol and enalapril in the present of enalaprilat in human plasma. Int J Appl Pharm 2018;10:31-40.

11. Bhardwaj SP, Singh S. Study of the forced degradation behavior of enalapril maleate by LC and LC-MS and development of a validated stability-indicating assay method. J Pharm Biomed Anal 2008;46:113-20. 